\title{
Etiology of small bowel thickening on computed tomography
}

\author{
Lee Finkelstone $M D^{1}$, Ellen Wolf $M D^{1}$, Marjorie W Stein $M D^{2}$
}

\begin{abstract}
L Finkelstone, E Wolf, MW Stein. Etiology of small bowel thickening on computed tomography. Can J Gastroenterol 2012;26(12):897-901.

BACKGROUND: Abdominal pain is often evaluated using imaging, most often with computed tomography (CT). While CT is sensitive and specific for certain diagnoses, small bowel thickening is a nonspecific finding on CT with a broad differential diagnosis including infection, inflammation, ischemia and neoplasm.

METHOD: A review of medical records of patients who underwent CT scans of the abdomen and pelvis over a one-year period and exhibited small bowel thickening were retrospectively evaluated to determine the final diagnosis.

RESULTS: The etiologies of small bowel thickening on CT were as follows: infection (113 of 446 [25.34\%]); reactive inflammation (69 of $446[15.47 \%]$ ); primary inflammation (62 of $446[13.90 \%])$; small bowel obstruction (38 of 446 [8.52\%]); iatrogenic (33 of 446 [7.40\%]); neoplastic (32 of 446 [7.17\%]); ascites (30 of 446 [6.73\%]); unknown (28 of $446[6.28 \%])$; ischemic (24 of $446[5.38 \%])$; and miscellaneous (17 of $446[3.81 \%])$.

CONCLUSION: Infectious and inflammatory (primary or reactive) conditions were the most common cause of small bowel thickening in the present series; these data can be used to formulate a more specific differential diagnosis.
\end{abstract}

Key Words: Inflammatory; Ischemic; Neoplastic; Small bowel; Thickening

mall bowel thickening is a common but nonspecific finding apparent on abdominal computed tomography (CT) and small bowel series in patients with abdominal pain and gastrointestinal symptoms. Differentiation of the various causes of small bowel thickening using these imaging studies is often not feasible but is important in providing effective patient care. The radiological literature has attempted to narrow the differential diagnosis by correlating various imaging features, such as the degree and distribution of wall thickening and mural enhancement patterns, with the specific etiology of wall thickening. However, none of these imaging patterns are, by themselves, diagnostic. The referring physician is often faced with a radiology report citing a long list of differential diagnoses for small bowel thickening and 'clinical correlation' may be recommended. The clinician must then correlate the history, physical examination, laboratory values, endoscopy or other studies with the vast differential diagnosis provided by the radiologist. The aim of the present study was to close a gap in the radiological literature by identifying the frequency - not the radiological pattern - of various causes of small bowel thickening in a large series of patients in an urban, poor, inner-city environment.

\section{METHODS}

A total of 32,896 CT scans and 199 small bowel series (SBS) were performed at the Montefiore Medical Center, a single, large urban medical centre located in New York (USA), from January to December 2008. Using the Radiology Information Systems-IC database, all final

\section{L'étiologie de l'épaississement de l'intestin grêle à la tomodensitométrie}

HISTORIQUE : Les douleurs abdominales sont souvent évaluées par imagerie, en général par tomodensitométrie (TD). La TD est sensible et spécifique dans certains diagnostics, mais l'épaississement de l'intestin grêle est une observation non spécifique à la TD et s'associe à un vaste diagnostic différentiel, y compris l'infection, l'inflammation, l'ischémie et le néoplasme.

MÉTHODOLOGIE : Les chercheurs ont procédé à l'analyse rétrospective des dossiers médicaux des patients qui ont subi une TD de l'abdomen et du bassin pendant une période d'un an et présenté un épaississement de l'intestin grêle afin de déterminer le diagnostic définitif.

RÉSULTATS : Les étiologies de l'épaississement de l'intestin grêle à la TD s'établissaient comme suit : infection (113 cas sur 446 [25,34\%]), inflammation réactive (69 cassur 446 [15,47\%]), inflammation primaire (62 cas sur $446[13,90 \%])$, obstruction de l'intestin grêle (38 cas sur $446[8,52 \%])$, atteinte iatrogène (33 cas sur $446[7,40 \%])$, atteinte néoplasique (32 cas sur $446[7,17 \%]$ ), ascites (30 cas sur 446 [6,73\%]), origine inconnue (28 cas sur 446 [6,28\%]), atteinte ischémique (24 cas sur 446 [5,38 \%]) et atteintes diverses (17 cas sur 446 [3,81\%]).

CONCLUSION : Dans la présente série, les maladies infectieuses et inflammatoires (primaires ou réactives) étaient les principales causes d'épaississement de l'intestin grêle. On peut utiliser ces données pour formuler un diagnostic différentiel plus précis.

radiology reports were searched for the word "thickening". A total of 446 patients who underwent CT scans, 28 patients who underwent SBS and five patients who underwent both studies within one month of one another demonstrating small bowel thickening and whose clinical charts were available were identified.

There were 251 women and 195 men in the CT group with a mean $( \pm$ SD) age of $54 \pm 19.6$ years. There were 10 women and 18 men in the SBS group with a mean age of $40.7 \pm 18.6$ years. The radiology reports were reviewed for anatomical location (duodenum, jejunum, ileum, multiple sites, no site specified) and distribution (diffuse versus focal) of small bowel thickening. If a specific site of small bowel wall thickening was not mentioned in the final report, the location was categorized as 'no site specified', but not duodenal because it is departmental practice to specifically mention duodenal thickening if present. The images were not reviewed at the time of the analysis unless there was a statement in the radiology report that questioned whether the apparent small bowel thickening was due to nondistension. In those cases, the images were reviewed by two board-certified radiologists with more than 20 years experience in gastrointestinal radiology, with differences resolved by consensus. Cases in which the bowel was deemed nondistended on image review were excluded from the study. The original reports were reviewed by five board-certified fellowship-trained body imagers with between five and 25 years of experience. In our practice, normal small bowel wall thickness is defined as indiscernible to $1 \mathrm{~mm}$ to $2 \mathrm{~mm}$ thick, and small bowel thickening as $>3 \mathrm{~mm}$.

\footnotetext{
${ }^{1}$ Albert Einstein College of Medicine, Montefiore Medical Center; ${ }^{2}$ Department of Radiology, Montefiore Medical Center, Bronx, New York, USA

Correspondence: Dr Marjorie W Stein, Department of Radiology, Montefiore Medical Center, 111 East 210th Street, Bronx, New York 10467, USA.

Telephone 718-920-6130, e-mail mstein17@aol.com.

Received for publication May 3, 2012. Accepted May 12, 2012
} 
TABLE 1

Etiology of small bowel thickening on computed tomography $(n=446)$

\begin{tabular}{lc}
\hline Etiology & Patients, $\mathbf{n}$ (\%) \\
\hline Infection & $113(25.3)$ \\
Reactive inflammatory & $69(15.5)$ \\
Primary inflammatory & $62(13.9)$ \\
Small bowel obstruction & $38(8.5)$ \\
latrogenic & $33(7.4)$ \\
Neoplastic & $32(7.2)$ \\
Ascites & $30(6.7)$ \\
Unknown & $28(6.3)$ \\
Ischemia & $24(5.4)$ \\
Miscellaneous & $17(3.8)$ \\
\hline
\end{tabular}

A retrospective chart review was then performed to determine the etiology of small bowel thickening in each case. The electronic patient file chart was used to analyze the discharge diagnosis, physician's notes, laboratory values, colonoscopy/endoscopy reports and pathology results to identify the final diagnosis in each case. The diagnosis was sorted into 10 different diagnostic categories: infectious, reactive inflammatory, primary inflammatory, small bowel obstruction (SBO), iatrogenic, neoplastic, ascites, ischemic, miscellaneous and unknown. Although many of the patients' histories were quite complex, they were classified into only one diagnostic category based on their final discharge diagnosis and clinical course. The final diagnosis for the etiology of small bowel wall thickening in the study population was calculated overall and followed by a subgroup analysis depending on the specific location of the small bowel affected. The etiologies of small bowel thickening in the duodenum were compared with the etiologies in patients without duodenal involvement to determine whether there were any significant differences. Finally, a comparison was made among five patients who underwent both a CT scan and SBS within one month of one another to correlate the two studies. The institutional review board approved the study and patient informed consent was not required.

Criteria for the infectious category included documentation of the diagnosis by culture or successful treatment for an infectious enteritis, routine blood work (white blood cell count $>10.8 \times 10^{9} / \mathrm{L}$, left shift) and stool studies. Criteria for the reactive inflammatory category included a primary diagnosis of abdominal or pelvic pathology that led to a secondary reactive inflammatory process in the adjacent small bowel. These diagnoses were confirmed with either pathology or a definite clinical diagnosis with successful treatment. Inclusion in the primary inflammatory category required a primary diagnosis of an inflammatory condition of the small bowel such as Crohn disease or peptic ulcer disease. The diagnosis of SBO required the presence of dilated proximal small bowel with a transition point to decompressed bowel distally on imaging and corroboration with the clinical findings. Criteria for the iatrogenic category required a definitive antecedent etiology, such as a procedure, surgery, medication or radiation. For the neoplastic category, a new or known malignancy was documented. Inclusion in the ascites category required significant ascites and no other documented small bowel or intra-abdominal pathology. The ischemia category required documentation of clinical or radiographic diagnosis of ischemic bowel. The miscellaneous category included diagnoses that did not fit into any of the other categories. The unknown category included patients in whom a definitive discharge diagnosis was not made and also included those who signed out against medical advice, expired from another medical problem, were lost to follow-up, or the patient's symptoms resolved spontaneously and no further diagnostic procedures were performed.

CT scans were performed using a 64 detector-row HiSpeed Advantage Scanner (GE Healthcare, USA) or 16 detector-row MD 1600 (Phillips, Netherlands). Scans were obtained using intravenous

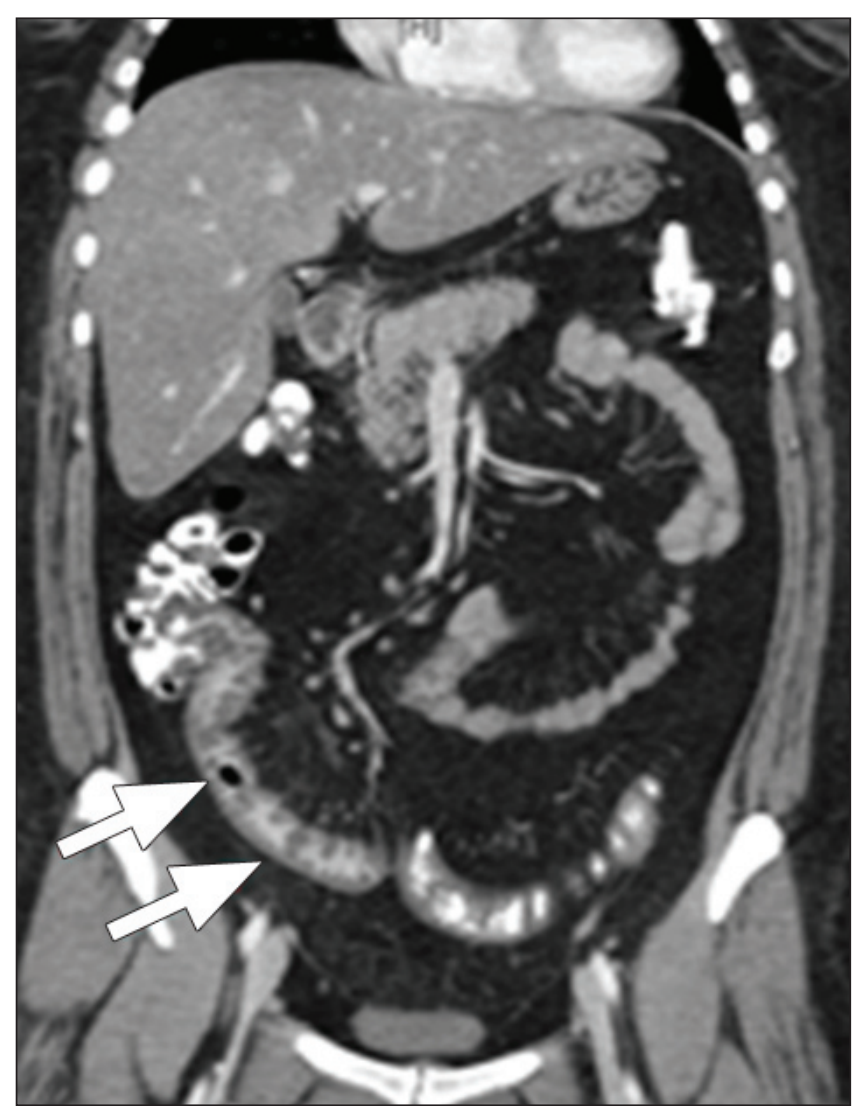

Figure 1) Computed tomography scan of a 34-year-old woman with documented infectious ileitis secondary to tuberculosis and ileal wall thickening (arrows)

administration of iohexol 300 (Omnipaque 300, Sanofi-Winthrop, USA) or iopromide 300 (Schering, Germany) administered using a single uniphasic bolus of $100 \mathrm{~mL}$ to $140 \mathrm{~mL}$ via a power injector at a rate of $2 \mathrm{~mL} / \mathrm{s}$ to $3 \mathrm{~mL} / \mathrm{s}$. Intravenous contrast was not administered if there was a contraindication to its use, which was the case in 112 patients (25\%). Routine CT of the abdomen and pelvis was performed in the portal-venous phase, with a $70 \mathrm{~s}$ to $80 \mathrm{~s}$ delay, a pitch of between 1.2 and 1.5 , and collimation ranging from $3 \mathrm{~mm}$ to $5 \mathrm{~mm}$. Oral contrast was routinely administered using $800 \mathrm{~mL}$ of $2 \%$ gastografin $45 \mathrm{~min}$ to $60 \mathrm{~min}$ before scanning.

The Fisher's exact test was used for statistical analysis and $\mathrm{P}<0.05$ was considered to be statistically significant.

\section{RESULTS}

A total of 462 patients comprised the initial study group. After image review, 16 patients were deemed to have underdistension of the bowel and not true small bowel thickening and, therefore, were excluded from the study. Of the remaining 446 patients, small bowel thickening was present in the following anatomical locations: 144 in the ileum; 86 in the duodenum; 43 in the jejunum; 19 with diffuse small bowel thickening; six had both duodenal and jejunal thickening; and two had both jejunal and ileal thickening. One hundred sixty-two patients did not have a specific site of small bowel thickening documented in their radiology reports.

Of the 446 patients who comprised the study group, the majority of patients with small bowel thickening on CT had infectious, or reactive inflammatory or primary inflammatory etiologies (Table 1).

Of the 113 patients with an infectious etiology, 30 (26.5\%) had specific organisms identified and documented (Figure 1). The organisms were: Helicobacter pylori ( $\mathrm{n}=11)$, Mycobacterium tuberculosis $(\mathrm{n}=5)$, Candida albicans $(\mathrm{n}=3)$, Clostridium difficile $(\mathrm{n}=2)$ and one each had Streptococcus bovis, Bacteroides, Escherichia coli, Proteus, Mycobacterium 


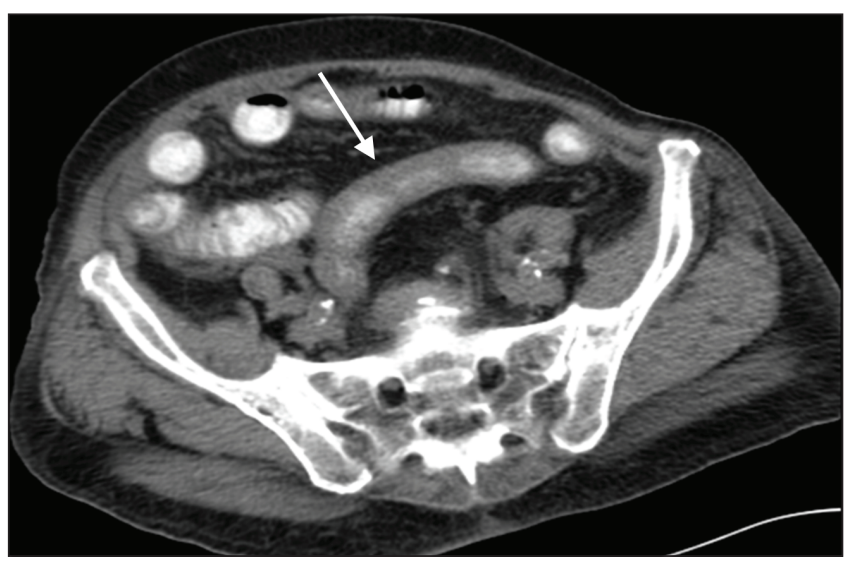

Figure 2) Computed tomography scan demonstrating jejunal thickening secondary to infection with no organism isolated (arrow) in an 81-year-old woman

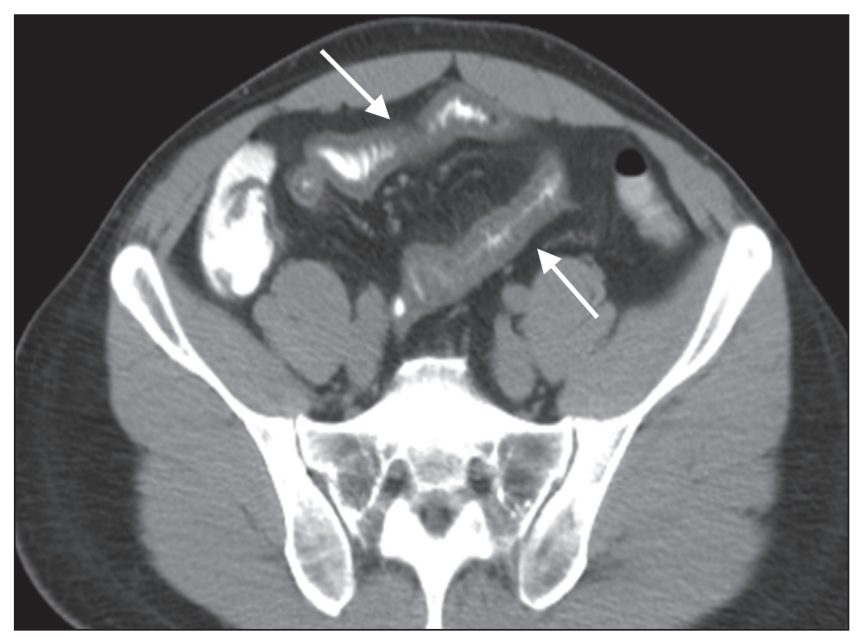

Figure 3) Computed tomography scan demonstrating ileal thickening secondary to Crohn disease (arrows) in a 39-year-old man

avium intracellularae, Mycobacterium avium complex, Pneumocystis jiroveci, Serratia and Staphylocoocus aureus. Of the remaining patients, 67 had an elevated white blood cell count or left shift that supported the clinical diagnosis of infectious enteritis. There were 16 patients who did not have a documented organism or blood work to support the clinical diagnosis; however, they underwent successful medical treatment, usually with antibiotics and, were therefore, presumed to have experienced an infectious enteritis (Figure 2).

Reactive inflammatory processes included appendicitis in 27 patients, pancreatitis in 17, diverticulitis in nine, pelvic inflammatory disease and other gynecological pathology, such as ruptured ovarian cyst, in 10 and cholecystitis in six.

Of the 62 patients in the primary inflammatory category, $27 \mathrm{had}$ Crohn disease, 20 had nonspecific inflammatory duodenitis or peptic ulcer disease, and six had nonspecific inflammatory ileitis (Figure 3). Three patients each had nonspecific inflammatory jejunitis, chronic nonspecific small bowel inflammation and small bowel diverticulitis.

Of the 33 patients in the iatrogenic category, 21 experienced postsurgical reactive changes and/or complications, seven experienced postprocedural complications, and five chemotherapy and/or radiation changes.

Of the 32 patients with neoplasms, there were 13 adenocarcinomas: six primary ileal, four duodenal and three jejunal. There was one carcinoid tumour, six cases of lymphoma and three cases of pancreatic carcinoma invading adjacent small bowel. Ten patients had metastatic disease from stomach, cervix, prostate and breast primaries.
TABLE 2

Etiology of small bowel thickening on computed tomography in the duodenum versus nonduodenal small bowel

\begin{tabular}{lccc}
\hline & \multicolumn{2}{c}{ Thickening, $\mathbf{n}(\%)$} & \\
\cline { 2 - 3 } Etiology & $\begin{array}{c}\text { In duodenum } \\
\text { only (n=86) }\end{array}$ & $\begin{array}{c}\text { Not in duodenum } \\
\text { ( } \mathbf{n = 3 6 0 )}\end{array}$ & $\mathbf{P}$ \\
\hline Infection & $17(19.8)$ & $96(26.7)$ & 0.22 \\
Reactive inflammatory & $20(23.3)$ & $49(13.6)$ & 0.03 \\
Primary inflammatory & $16(18.6)$ & $46(12.8)$ & 0.17 \\
Small bowel obstruction & $1(1.2)$ & $37(10.3)$ & 0.004 \\
Ascites & $1(1.2)$ & $29(8.1)$ & 0.017 \\
latrogenic & $8(9.3)$ & $25(6.9)$ & 0.49 \\
Neoplastic & $9(10.5)$ & $23(6.4)$ & 0.24 \\
Ischemic & $3(3.5)$ & $21(5.8)$ & 0.59 \\
Unknown & $9(10.5)$ & $19(5.3)$ & 0.08 \\
Miscellaneous & $2(2.2)$ & $15(4.2)$ & 0.54 \\
\hline
\end{tabular}

TABLE 3

Etiology of small bowel thickening on computed tomography in the jejunum versus the ileum

\begin{tabular}{lccc}
\hline & \multicolumn{2}{c}{ Thickening, $\mathbf{n}(\%)$} & \\
\cline { 2 - 3 } Etiology & $\begin{array}{c}\text { In jejunum only } \\
(\mathbf{n}=\mathbf{4 3})\end{array}$ & $\begin{array}{c}\text { In ileum only } \\
(\mathbf{n}=\mathbf{1 4 4 )}\end{array}$ & $\mathbf{P}$ \\
\hline Infection & $9(20.9)$ & $44(26.7)$ & 0.25 \\
Reactive inflammatory & $6(14.0)$ & $25(13.6)$ & 0.81 \\
Primary inflammatory & $0(0.0)$ & $29(12.8)$ & 0.0005 \\
Small bowel obstruction & $6(14.0)$ & $12(10.3)$ & 0.038 \\
Ascites & $0(0.0)$ & $0(8.1)$ & - \\
latrogenic & $0(0.0)$ & $0(6.9)$ & - \\
Neoplastic & $0(0.0)$ & $8(6.4)$ & 0.20 \\
Ischemic & $5(11.6)$ & $0(5.8)$ & 1.00 \\
Unknown & $0(10.5)$ & $10(5.3)$ & 0.12 \\
Miscellaneous & $6(14.0)$ & $0(4.2)$ & 0.0001 \\
\hline
\end{tabular}

Of the 30 patients with significant ascites, 26 had liver failure, two had ascites of unknown etiology and one each had severe heart failure and severe renal failure.

Of the 446 patients, 360 exhibited small bowel thickening not involving the duodenum. Of these 360 patients, the majority had small bowel thickening due to infectious, reactive inflammatory or primary inflammatory etiologies (Table 2). Of the 86 patients with only duodenal thickening, the majority were due to infectious, reactive inflammatory or primary inflammatory etiologies (Table 2). Of the 20 patients with reactive inflammatory etiologies in the duodenal thickening group, the majority (13 of 20 [65\%]) were due to pancreatitis. In the 17 patients with infectious etiologies in the duodenum, 11 had documented organisms, the most common of which was $H$ pylori in seven (63.63\%). Statistically significant differences between patients with and without duodenal involvement were apparent only in the reactive inflammatory, $\mathrm{SBO}$ and ascites groups (Table 2).

Of the 144 patients with ileal thickening, the majority had infectious, primary inflammatory processes (most commonly Crohn disease) and reactive inflammatory conditions (most commonly appendicitis) (Table 3). Of the 44 patients with ileal thickening due to infection, four had documented organisms including Candida $(\mathrm{n}=2)$, Serratia $(n=1)$ and mixed flora $(n=1)$.

Of the 43 patients who exhibited jejunal thickening only, the most common etiologies included infection, SBO, reactive inflammatory and miscellaneous (Table 3). Of the nine patients with jejunal thickening due to infection, three had documented organisms and all had H pylori. 


\begin{tabular}{lc}
$\begin{array}{l}\text { TABLE } \mathbf{4} \\
\text { Etiology of small bowel thickening on small bowel series } \\
(\mathbf{n}=\mathbf{2 8})\end{array}$ & Patients, $\mathbf{n}(\%)$ \\
\hline Etiology & $16(57.1)$ \\
\hline Primary inflammatory & $7(25.0)$ \\
Unknown & $2(7.1)$ \\
Neoplastic & $1(3.6)$ \\
Small bowel obstruction & $1(3.6)$ \\
Reactive inflammatory & $1(3.6)$ \\
\hline Infection &
\end{tabular}

Of the 19 patients with diffuse thickening, the majority had ascites secondary to liver failure.

There were significantly fewer SBS $(n=199)$ than CT scans $(n=32,896)$ performed during this time period and only 28 that reported small bowel thickening. Of these 28 patients, the clinical diagnoses in the majority were due to primary inflammatory etiologies (Table 4). Of the 16 patients with a primary inflammatory etiology, nine had inflammatory duodenitis and seven had Crohn disease. The malignancies included gallbladder carcinoma with duodenal involvement and desmoplastic round cell tumour. The one infectious etiology was due to $M$ avium intracellulare. The etiology of reactive inflammatory disease was pancreatitis. The anatomical location of small bowel thickening on the 28 SBS included the duodenum $(n=18)$, the ileum $(n=8)$, the jejunum $(n=1)$ and no site specified $(n=1)$.

There were only five patients in the present study who had documented small bowel thickening on CT scan and an SBS performed within one month of one another. Three of the five patients had Crohn disease, one had inflammatory duodenitis and one had gastroenteritis. The CT scans and SBS were concordant in the three Crohn disease patients but discordant in the other two patients in whom the SBS was normal.

\section{DISCUSSION}

Small bowel thickening is a relatively nonspecific finding on CT with a broad differential diagnosis. Our study demonstrated that infectious and inflammatory etiologies are the most frequent causes of small bowel thickening. Previous studies have focused on differentiating true small bowel thickening from underdistended normal small bowel. In a distended small bowel loop, normal wall thickness should be indiscernible to $1 \mathrm{~mm}$ to $2 \mathrm{~mm}$ thick. If the bowel is underdistended, then the wall may measure up to $2 \mathrm{~mm}$ to $3 \mathrm{~mm}$ thick. There is controversy in the literature because some authors believe that the criterion for the upper limit of thickness in normally distended bowel is $2 \mathrm{~mm}$ to $3 \mathrm{~mm}$, while others believe that any discernible wall thickening indicates pathology (1-7). One approach used to differentiate pathological small bowel thickening from underdistended normal segments is to compare wall thickness between similarly distended bowel segments, but this may be difficult when diffuse pathology is present.

Other studies have analyzed specific radiological features, such as degree of wall thickening, distribution (focal, segmental and diffuse), attenuation and enhancement patterns, and pathology outside the gastrointestinal tract to distinguish between benign and malignant etiologies. However, none of these individual CT findings are, by themselves, diagnostic in all cases, and there is significant overlap in the radiological appearance of various entities (1-15). Often, the specific etiology remains inconclusive, thus requiring clinical correlation and additional studies for a definitive diagnosis, increasing patient anxiety and medical costs. Our large study was novel and addresses a gap in the literature because we determined the relative frequencies of causes of small bowel thickening - an approach, which to our knowledge, has not been published previously. This epidemiological information could be used as an additional tool to help narrow the differential diagnosis of small bowel thickening on CT scan.

Although the specific organism was not identified in most patients with infection, the most common organism isolated was $H$ pylori, with the majority located in the duodenum. The most common conditions in our study causing reactive inflammatory thickening were appendicitis and pancreatitis. The most common conditions causing primary inflammatory thickening was Crohn disease and nonspecific enteritis.

Statistically significant differences in the patient groups with and without duodenal involvement were in the categories of reactive inflammatory, SBO and ascites. Reactive inflammatory etiologies were statistically more common in the duodenal group. This can be explained by the proximity of the pancreas to the duodenum in the patients with pancreatitis, who comprised the majority of cases of duodenal thickening in the reactive inflammatory group. As a cause of small bowel thickening, $\mathrm{SBO}$ was more common in patients without duodenal involvement because most sites of obstruction occur in the more distal small bowel. Ascites as a cause of small bowel thickening is more likely to affect nonduodenal small bowel due to the often diffuse distribution of ascitic fluid.

The differences in etiologies of small bowel thickening in the jejunal and ileal groups were only statistically significant in the primary inflammatory group. This was due to the predilection for Crohn disease in the ileum.

Other diagnostic categories, including neoplastic, ischemic, $\mathrm{SBO}$ and ascites, were less prevalent. These conditions often have ancillary radiological evidence to help support the diagnosis, whether it is asymmetric $>2 \mathrm{~cm}$ wall thickening with malignancy, the presence of an embolus or thrombus with ischemia, a transition point in SBO or a cirrhotic liver with ascites. Thus, in the absence of supportive findings such as these and, given that infectious and inflammatory etiologies are statistically the most common etiologies, one can be more confident in narrowing the differential diagnosis.

With regard to SBS, perhaps the most significant observation was the small number of studies that were performed during this one-year period. Furthermore, the patients in this group were, on average, younger than those in the CT group. After the CT scan and laboratory work was performed, clinicians often proceeded directly to endoscopy, bypassing the SBS. Determining the underlying etiology for many of the SBS was more challenging than the CT scans due to less documentation and more remote follow-up. Thus, significant conclusions about underlying etiologies on SBS are difficult to draw. Future studies of SBS would require a longer time period with a larger sample of patients with small bowel thickening noted on SBS to establish any meaningful conclusions.

Our study has several limitations. Because our study was retrospective in nature, the clinical information that was gathered from patient charts may have been incomplete. Although many of the patients' histories were complex, they were classified into only one diagnostic category based on discharge diagnosis and clinical course. Pathological confirmation from many patients was lacking.

Our institution is a large, tertiary care hospital in the inner-city of a large metropolis. Our patients are mainly poor and Hispanic, and many suffer from major health problems such as obesity, hypertension and diabetes. This was a strength of our study because we focused on an often under-reported population in the literature. However, a potential limitation of our study is that our demographics may not necessarily be extrapolated to all populations.

\section{SUMMARY}

Small bowel thickening on CT scan is a nonspecific radiological finding but is most often of infectious or inflammatory etiology. The radiologist can use this frequency data as an additional tool to help formulate a more specific differential diagnosis. 


\section{REFERENCES}

1. Macari M, Blathazar EJ. CT of bowel wall thickening: Significance and pitfalls of interpretation. AJR 2001;176:1105-16.

2. Balthazar EJ. CT of the gastrointestinal tract: Principles and interpretation. AJR 1991;56:23-32.

3. Shirkhoda A. Diagnostic pitfalls in abdominal CT. RadioGraphics 1991;11:969-1002.

4. Gore RM, Balthazar EJ, Ghahremani GG, Miller FH. CT features of ulcerative colitis and Crohn's disease. AJR 1996;167:3-15.

5. James S, Balfe DM, Lee JKY, Picus D. Small bowel disease: Categorization by CT examination. AJR 1987;148:863-8.

6. Scanlon MH, Blumberg ML, Ostrum BJ. Computed tomographic recognition of gastrointestinal pathology. RadioGraphics 1982;3:201-27.

7. Karahan OI, Dodd GD III, Chintapalli KN, Rhim H, Chopra S. Gastrointestinal wall thickening in patients with cirrhosis: Frequency and patterns at contrast-enhanced CT. Radiology $2000 ; 215: 103-7$.
8. Hoon J, Pablo RR. Spiral CT of the small bowel. Applied Radiol 1999;28:11-5.

9. Wiesner W, Khurana B, Hoon J, Pabo RR. CT of acute bowel ischemia. Radiology 2003;226:635-50.

10. Chou CK. CT manifestations of bowel ischemia. AJR 2002;178:87-91.

11. Kalantari BK, Mortele KJ, Cantisani V, et al. CT features with pathologic correlation of acute graft-versus-host disease after bone marrow transplantation in adults. AJR 2003;181:1621-5.

12. Gelfand DW, Ott DJ, Chen MYM. Radiologic evaluation of gastritis and duodenitis. AJR 1999;173:357-61.

13. Jacobs JM, Hill MC, Steinberg WM. Peptic ulcer disease: CT evaluation. Radiology 1991;178:745-8.

14. Sandrasegaran K, Maglinte DD, Lappas JC, Howard TJ. Small-bowel complications of major gastrointestinal tract surgery. AJR 2005;185:671-81.

15. Freeman AH. CT and bowel disease. Br J Radiol 2001;74:4-14. 


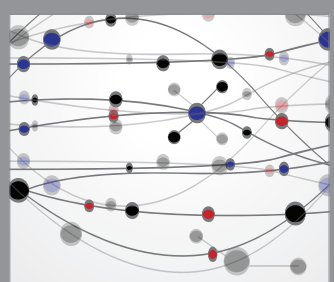

The Scientific World Journal
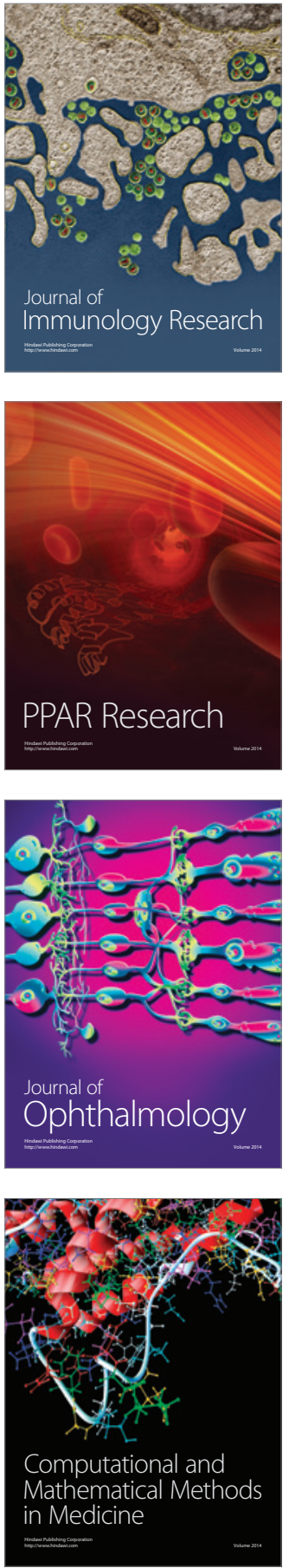

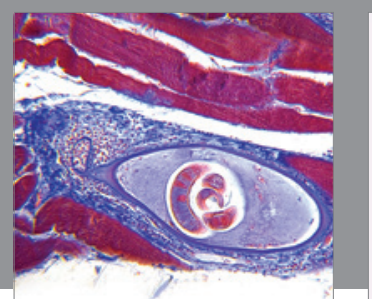

Gastroenterology Research and Practice

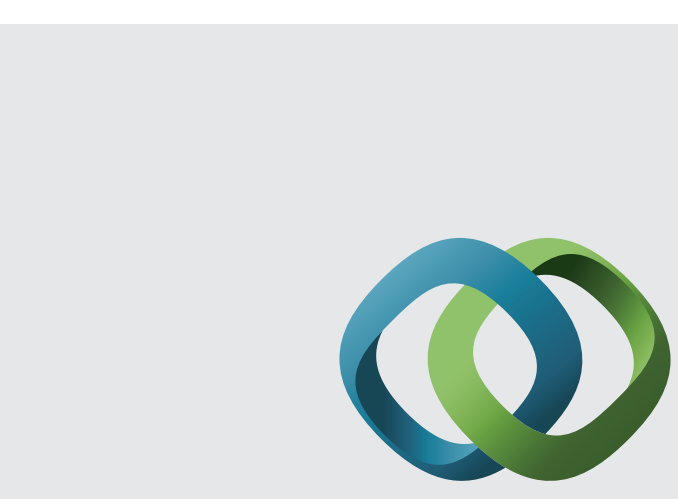

\section{Hindawi}

Submit your manuscripts at

http://www.hindawi.com
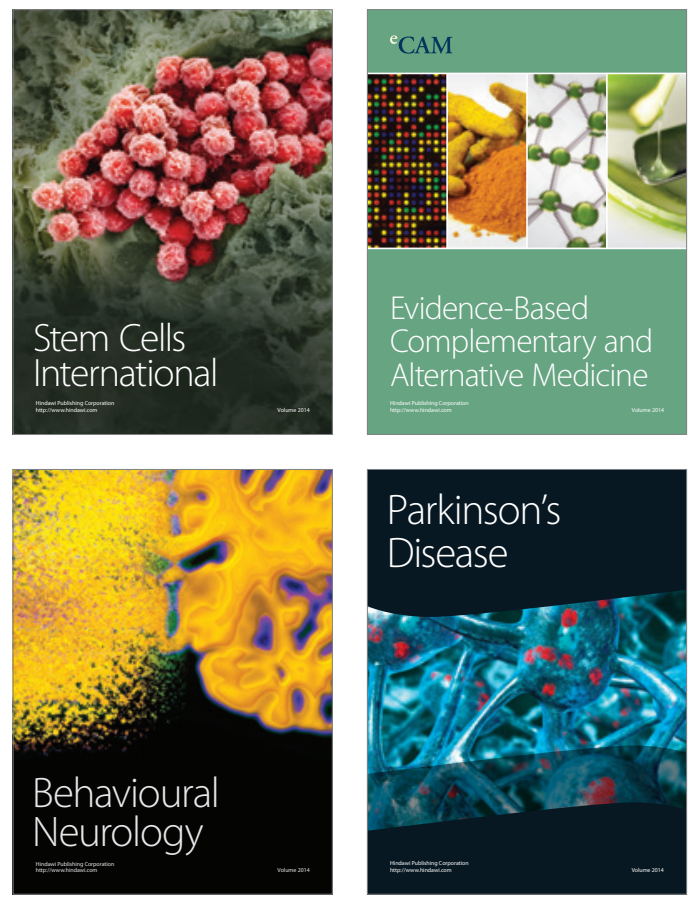
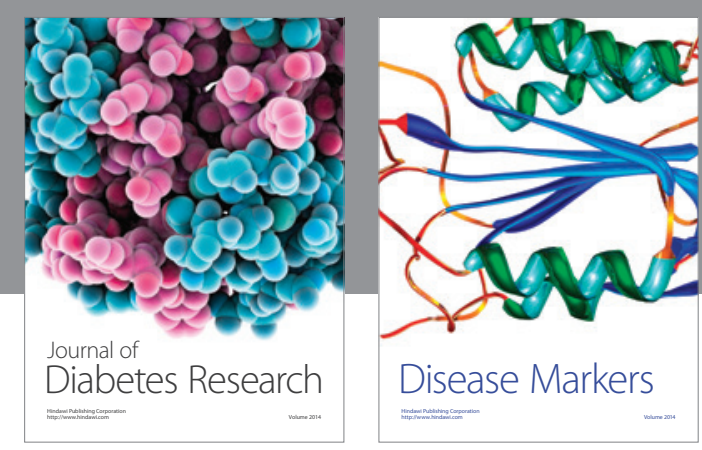

Disease Markers
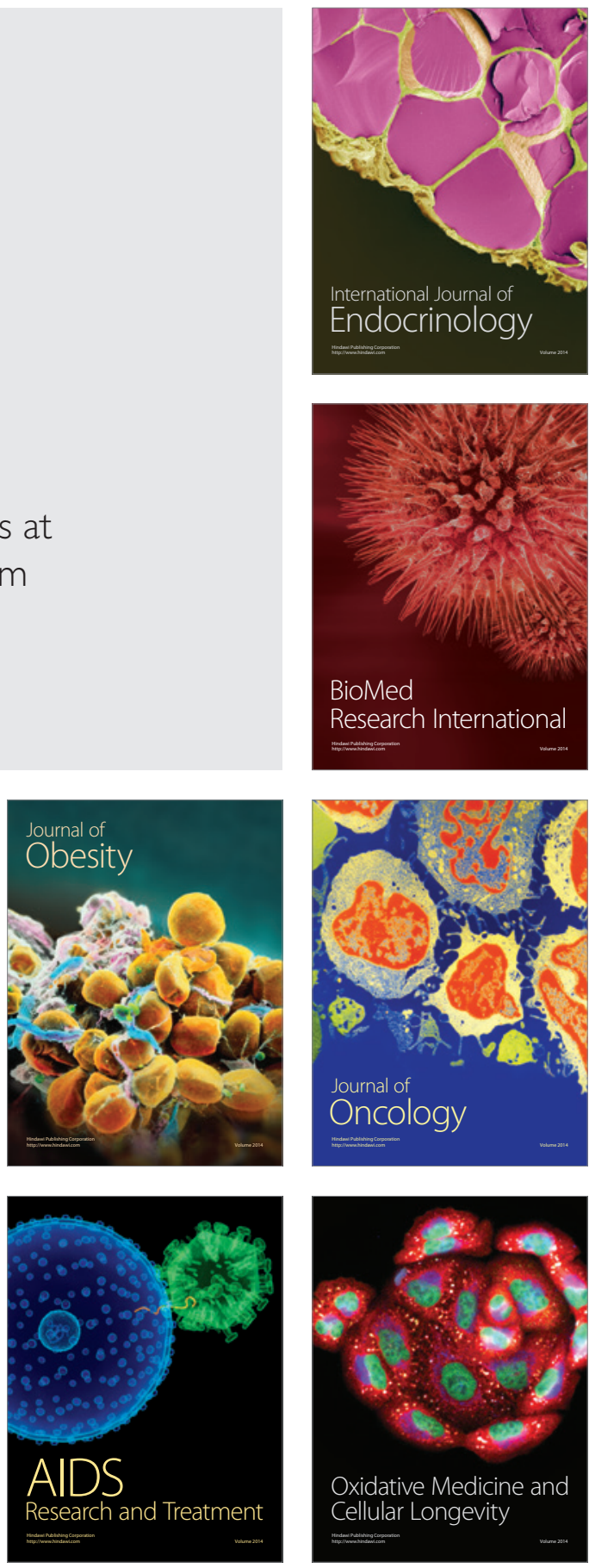